\title{
EDITORIAL
}

\section{Difference between hematological malignancy and Solid tumor research articles published in four major medical journals}

Leukemia (2006) 20, 1655-1657. doi:10.1038/sj.leu.2404369

Hematological malignancies are rare malignant disorders. Deaths from 'lymphoid, hematopoietic and related tissues' (C81-96 by ICD-10) accounts merely for $6.2 \%$ of all deaths from malignancies. ${ }^{1}$ However, basic research and the development of new treatments based on results of basic research are thought to be more advanced in hematological malignancies than in solid tumors, as specimens of malignant cells can be obtained easily from peripheral blood of patients with hematological malignancies, particularly with leukemia. Thus, general clinicians may be more frequently exposed to research results on hematological malignancies than on solid tumors. However, no study has reported on the effect of different nature of the primary diseases on the frequency of publications.

In major clinical journals, 8707 research articles were published between 1995 and 2004, which were 3035 in the Lancet, 2147 in the New England Journal of Medicine (NEJM), 1199 in Annals of Internal Medicine (Ann Intern Med), and 2326 in Journal of the American Medical Association (JAMA). Among them, 1118 articles (12.8\%) studies about malignancies: 200 on hematological malignancies, 824 on solid tumors and 94 on combined malignancies. Figure 1 shows the number of research articles on malignancies between 1995 and 2004. No significant year-to-year difference was observed, except for 2001.

Among 1118 articles on malignancies, 60 (5\%) were on basic researches and 1058 (95\%) were on clinical researches. The

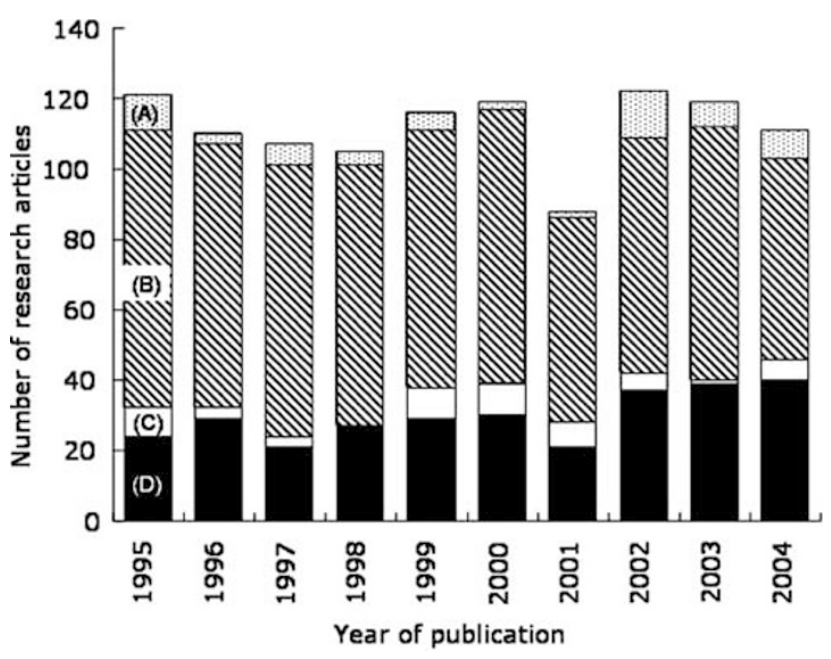

Figure 1 Numbers and categories of research articles on malignancies between 1995 and 2004. Although the yearly numbers of research articles between 1995 and 2004 have not significantly changed, the number of observational studies declined and the number of clinical trials, particularly randomized trials, are on the increasing trend. (a) Basic researches, (b) clinical researches: observational studies, (c) clinical trials without randomization and (d) clinical trials with randomization. clinical research articles contained 348 clinical trials and 710 observational studies. Of the clinical trials, 297 (85\%) were randomized. The observational studies contained 423 longitudinal ones (346 cohort and 77 case-control studies) and the other 287 studies. Figure 1 shows the categories of research articles between 1995 and 2004. The number of observational studies is declining, whereas clinical trials, particularly randomized trials, are increasing. Of the 1118 articles on malignancies, 294 (26\%) included hematological malignancies, among which 200 (18\%) focused on hematological malignancies. Figure 2 shows the numbers of research articles on hematological malignancies and solid tumors between 1995 and 2004. Basic researches were significantly more common in hematological malignancies than in solid tumors $(24 / 200(12 \%)$ vs $34 / 824(4.1 \%), P=0.0001$; Table 1$)$. Non-randomized clinical trials were more frequent in hematological malignancies than in solid tumors $(21 / 200$ (11\%) vs $28 / 324$ (3.4\%), $P=0.0006)$. Figure 3 shows the number of research articles on solid tumors, hematological malignancies and combined studies per 100 articles published in the four medical journals. The publication frequencies on solid tumors were not different among the four medical journals (7.8-10.9\%), whereas those on hematological malignancies were different. Hematological malignancies appeared most frequently in NEJM (4.8\%) and least frequently in JAMA (0.4\%). NEJM and the Lancet tended to frequently publish clinical trials, particularly randomized trials, both in solid tumors and hematological malignancies. On the contrary, JAMA and Ann Intern Med frequently published observational studies.

Malignancies have been a major topic in medical journals. Approximately 100 research articles are annually published in major clinical journals. In the four medical journals analyzed,

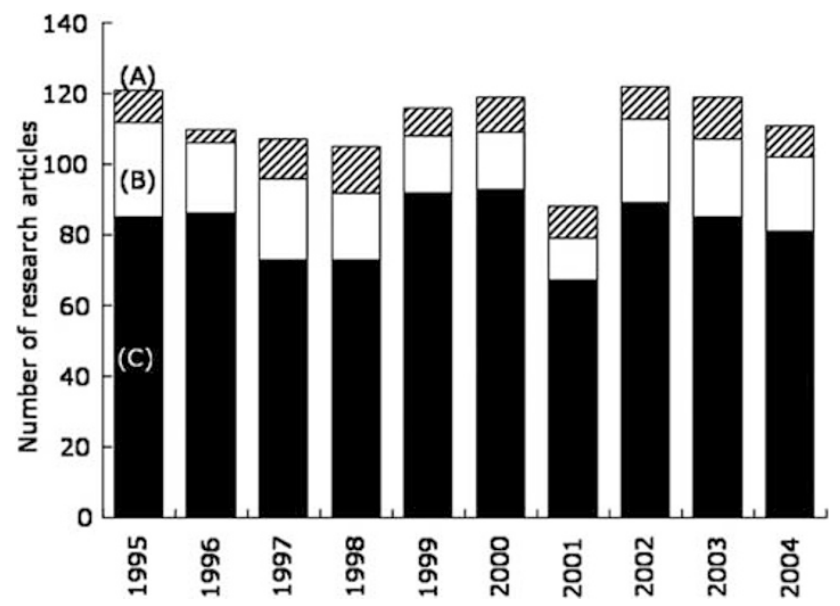

Figure 2 Numbers of research articles on hematological malignancies and solid tumors between 1995 and 2004. No significant change in the number of articles on hematopoietic and solid tumors is observed between 1995 and 2004. (a) Combined, (b) hematological malignancies and (c) solid tumors. 
Table 1 Comparison of research articles on hematological malignancies and solid tumors

\begin{tabular}{|c|c|c|c|c|c|c|}
\hline & & & & $\begin{array}{c}\text { Hematological } \\
\text { malignancies }\end{array}$ & $\begin{array}{l}\text { Solid } \\
\text { tumors }\end{array}$ & Combined \\
\hline $\begin{array}{l}\text { Total } \\
\text { Basic researches } \\
\text { Clinical researches }\end{array}$ & $\begin{array}{l}\text { Clinical trials } \\
\text { Observational studies }\end{array}$ & $\begin{array}{l}\text { Longitudinal studies } \\
\text { Others }\end{array}$ & $\begin{array}{l}\text { Randomized } \\
\text { Non-randomized } \\
\text { Cohort studies } \\
\text { Case-control studies }\end{array}$ & $\begin{array}{r}200 \\
24 \\
53 \\
21 \\
57 \\
7 \\
38\end{array}$ & $\begin{array}{r}824 \\
34 \\
231 \\
28 \\
252 \\
65 \\
214\end{array}$ & $\begin{array}{r}94 \\
2 \\
13 \\
2 \\
37 \\
5 \\
35\end{array}$ \\
\hline
\end{tabular}

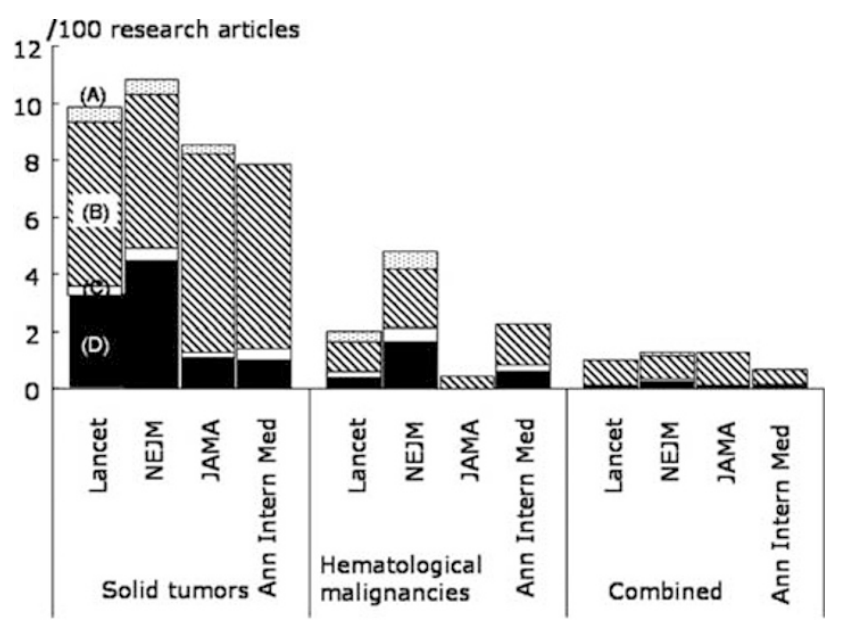

Figure 3 Numbers and categories of research articles on solid tumors and hematological malignancies per 100 articles in major clinical journals. Although the frequencies of publications on solid tumors were not significantly different among the four medical journals, publications on hematological malignancies were most common in NEJM (4.8\%) and least common in JAMA (0.4\%). NEJM and the Lancet tended to frequently publish clinical trials, particularly randomized trials, both in solid tumors and hematological malignancies. On the contrary, JAMA and Ann Intern Med frequently published observational studies. (a) Basic researches, (b) clinical researches: observation studies, (c) clinical trials without randomization and (d) clinical trials with randomization.

$10-15 \%$ of the published articles reported on malignancies. This tendency remains relatively stable throughout the study duration, 1995-2004. Aging of the population has recently been accelerated in developed countries and malignancies have surfaced as a social issue. A growing number of pharmaceutical companies have emphasized the development of anticancer agents. ${ }^{2}$ Given these backgrounds, the frequency of publications on malignancies is likely to increase in the near future.

These data revealed that the articles on hematological malignancies are frequently published in major medical journals, compared with the low prevalence. Although deaths from hematological malignancies accounts merely for $6.2 \%$ of deaths from malignancies in Japan, ${ }^{1} 26 \%$ of research articles on malignancies published in major clinical journals reported on hematological malignancies someway. Readers of major clinical journals have more opportunities to be exposed to information on hematological malignancies than that on solid tumors, despite the low prevalence of hematological malignancies. This fact is suggestive. The recent growth of medical specialization has brought about the need of cross-sectional coordination. Many clinicians consider following the major clinical journals such as those presented in this study beneficial to obtain information outside of their own specialties. However, little has reported that there is a certain tendency in topics published in these medical journals. Recognizing the publication tendency of the major clinical journals would be important for clinicians to acquire balanced medical knowledge.

Categories of published studies in hematological malignancies and solid tumors are significantly different (Table 1). Basic researches were more common in hematological malignancies than in solid tumors (12 vs $4.1 \%$ ). It is also characteristic of hematological malignancies that non-randomized clinical trials were common. These may be attributable to the nature of the diseases where the pathological mechanisms are frequently elucidated at a molecular level and to the sensitivity to anticancer drugs. Given the sensitivity, hematological malignancies may often be the first candidates in clinical trials on newly developed anticancer drugs and the first results reported in early phases of trials. Such examples are imatinib ${ }^{3,4}$ and bortezomib. ${ }^{5}$

These four major clinical journals differently present articles on hematological malignancies, in contrast with the similar frequencies in publications on solid tumors. These may reflect the difference in the target readers of each journal. NEJM is considered to be the most prestigious clinical journal and widely read by all medical professionals. JAMA and Ann Intern Med are published by the American Medical Association and the American College of Physicians, respectively, and the target readers of these journals may be different from those of NEJM. The frequent publications of randomized clinical trials which provide the highest evidence level in NEJM and the Lancet may have contributed to the high recognition of these journals.

In conclusion, the publications on hematological malignancies are more frequent than those on solid tumors despite the low prevalence; basic researches and non-randomized clinical trials are more common in hematological malignancies than in solid tumors; and hematological malignancies are presented differently among the major clinical journals. Clinicians would need to be aware of these tendencies of major clinical journals.

A Chizuka ${ }^{1}$, M Suda $^{2}$, T Shibata $^{3}$, E Kusumi $^{2}$, A Hori $^{2}$, T Hamaki $^{4}$, Y Kodama ${ }^{2}$, K Horigome $^{2}$, Y Kishi $^{5}, \mathrm{~K}$ Kobayashi $^{2}$, T Matsumura $^{2}, \mathrm{~K}$ Yuji ${ }^{2}, \mathrm{Y}$ Tanaka ${ }^{2}$ and $\mathrm{M} \mathrm{Kami}^{2}$

${ }^{1}$ Division of Hematology, Department of Medicine, Keio University School of Medicine, Tokyo, Japan;

${ }^{2}$ Division of Exploratory Research, Institute of Medical Science, University of Tokyo, Tokyo, Japan;

${ }^{3}$ Research Center for Cancer Prevention and Screening, National Cancer Center, Tokyo, Japan;

${ }^{4}$ Department of Transfusion Medicine, Metropolitan Fuchu Hospital, Tokyo, Japan and

${ }^{5}$ Division of Regenerative Medicine, Jichi Medical University, Tochigi, Japan

E-mail: kami-tky@umin.ac.jp 


\section{References}

1 Nomura K. Cancer Statistics in Japan-2005. Foundation for Promotion Cancer Research: Tokyo, 2005.

2 Featherstone J, Griffiths S. From the analyst's couch. Drugs that target angiogenesis. Nat Rev Drug Discov 2002; 1: 413-414.

3 Druker BJ, Sawyers CL, Kantarjian H, Resta DJ, Reese SF, Ford JM et al. Activity of a specific inhibitor of the BCR-ABL tyrosine kinase in the blast crisis of chronic myeloid leukemia and acute lymphoblastic leukemia with the Philadelphia chromosome. N Engl J Med 2001; 344: 1038-1042.

4 Druker BJ, Talpaz M, Resta DJ, Peng B, Buchdunger E, Ford JM et al. Efficacy and safety of a specific inhibitor of the BCR-ABL tyrosine kinase in chronic myeloid leukemia. N Engl J Med 2001; 344: 1031-1037.

5 Richardson PG, Barlogie B, Berenson J, Singhal S, Jagannath S, Irwin $\mathrm{D}$ et al. A phase 2 study of bortezomib in relapsed, refractory myeloma. N Engl J Med 2003; 348: 2609-2617. 